\title{
A AMP Agradece!
}

\section{AMP Says Thank You!}

Miguel GUIMARÃES ${ }^{1,2,3}$, Tiago VILLANUEVA $\bowtie{ }^{4,5}$

Acta Med Port 2022 Feb;35(2):79-83 - https://doi.org/10.20344/amp.4683

Entre 1 de janeiro e 31 de dezembro de 2021, a Acta Médica Portuguesa recebeu 1515 submissões através da sua plataforma electrónica Open Journal System. Ao longo do ano, os nossos serviços editoriais publicaram 209 artigos distribuídos pelas 12 edições regulares, e 115 artigos em ahead of print, o que equivale a mais sete edições. No total, foram publicadas 884 páginas, num esforço sem precedentes por parte de uma equipa dimensionada para a publicação de seis edições e para a gestão de menos de 500 submissões por ano.

Neste contexto, queremos em primeiro lugar agradecer aos Editores-Chefe Adjuntos, aos nossos Editores-Associados e à equipa in-house da Acta Médica Portuguesa, pelo extraordinário esforço e dedicação que mais uma vez demonstraram num período particularmente exigente e desafiante. Agradecemos ainda ao Miguel Reis, que ao longo de mais de 40 anos de exclusiva dedicação à AMP assegurou a sua continuidade. É com gratidão que realçamos o contributo inigualável do Miguel, que durante décadas foi a verdadeira "cara" da AMP, com todo o sacrifício pessoal e familiar inerente.

Decidir quais os trabalhos a publicar, procurando constituir uma mais-valia efectiva para os nossos leitores e assim contribuir para a promoção de boas práticas na investigação científica, para a melhoria da prática clínica, e para a divulgação do conceito da moderna autoria científica, é um processo complexo. Neste âmbito, a colaboração dos peritos a quem solicitámos a avaliação dos trabalhos propostos para publicação é fundamental. A Acta Médica Portuguesa é a única revista científica médica portuguesa de âmbito generalista indexada na MEDLINE, com uma audiência de mais de 45000 médicos portugueses, outros profissionais de saúde, decisores e população em geral. Como tal, é imprescindível o contributo de especialistas das várias áreas, que nos apoiam na identificação dos temas de maior relevância, comentam a pertinência dos estudos propostos, realçam as linhas inovadoras das metodologias apresentadas, etc.

Gostaríamos de salientar a importância de envolvermos mais médicos no processo de revisão por pares da "nossa" Acta Médica Portuguesa, independentemente da fase da carreira em que se encontram ou da sua especialidade. Só assim, todos juntos, alcançaremos o objectivo de consolidar a nossa posição enquanto referência nacional e até internacional. A inscrição na plataforma OJS pode ser feita rapidamente nesta página: https://www.actamedicaportuguesa.com/ revista/index.php/amp/user/register, e o input de todos quantos queiram arregaçar as mangas e ajudar-nos "a remar" nesta experiência única e enriquecedora será muitíssimo bem vindo.

A todos e a cada um de vós, que de forma contínua doam generosamente o vosso tempo, expressamos o sincero reconhecimento da Ordem dos Médicos e da Acta Médica Portuguesa..

Lisboa, 12 de Janeiro de 2022

\author{
Miguel Guimarães \\ Bastonário da Ordem dos Médicos \\ Tiago Villanueva \\ Editor-Chefe
}

A lista seguinte enumera os revisores que ao longo de 2021 procederam à avaliação de artigos a pedido da nossa publicação.

\footnotetext{
1. Bastonário. Ordem dos Médicos. Lisboa. Portugal.

2. Co-Editor. Acta Médica Portuguesa. Lisboa. Portugal.

3. Serviço de Urologia. Hospital de São João. Porto. Portugal.

4. Editor-Chefe. Acta Médica Portuguesa. Lisboa. Portugal.

5. Médico de Família. Unidade de Saúde Familiar Reynaldo dos Santos. Póvoa de Santa Iria. Portugal.

$\triangle$ Autor correspondente: Tiago Villanueva. tiago.villanueva@ordemdosmedicos.pt

Recebido: 12 de janeiro de 2021 - Aceite: 12 de janeiro de 2021 - Online issue published: 01 de fevereiro de 2022

Copyright $\odot$ Ordem dos Médicos 2022
} 
Revisores com oito avaliações concluídas:

Edgar Mesquita

Revisores com cinco avaliações concluídas:

Diogo Almeida

Marília Antunes

Revisores com quatro avaliações concluídas:

$\begin{array}{ll}\text { Álvaro Azevedo } & \text { Helena Sofia Antão } \\ \text { Ana Farinha } & \text { João Bernardes } \\ \text { Catarina Figueiredo Jacinto Correia } & \text { Micaela Antunes } \\ \text { Daniel Barrocas } & \text { Miguel Bigotte Vieira }\end{array}$

Paulo Cruz Paixão

Pedro Carreiro-Martins

Pedro Miguel Alves Ribeiro Correia

Revisores com três avaliações concluídas:

\author{
Ana Cardoso \\ Ana Rita Torres \\ Arminda Manuela Gonçalves \\ Carla André \\ David Marques Lito \\ Filipa Quaresma \\ Francisco Antunes \\ Guilherme Bastos Martins \\ Jessica Silva Lomba \\ Joana Almeida \\ Joana Teixeira \\ João Albuquerque Gonçalves
}

Joaquim Cerejeira
José Pedro Boléo-Tomé
Luís Agualusa
Manuel Bragança Pereira
Maria Céu Almeida
Maria Helena Pimentel
Maria João Aleixo
Maria Jorge Casanova
Maria Lopes Lima Alves
Maria Margarida Costa Ferreira Bandarra
Mariana Fragão-Marques
Meireles Brandão

Mónica Marçal

Nuno Abecasis

Paula Leiria Pinto

Paulo André Fernandes

Pedro Aguiar

Pita Barros

Raquel Esteves Marques

Ricardo Coentre

Sofia R. Valdoleiros

Teresa Bandeira

Zélia Anastácio

Revisores com duas avaliações concluídas:

\begin{tabular}{ll} 
Adalberto Campos Fernandes & Beatriz Minghelli \\
Adriana Belo Cabete & Beatriz Oliveira Pinto \\
Afonso Félix-Oliveira & Bruno Heleno \\
Alexandre Castro Caldas & Camila Marcelino Loureiro \\
Alexandre Gomes da Silva & Cândida Cancelinha \\
Alice Coimbra & Carla Gomes da Silva \\
Amélia Almeida & Carla Viegas \\
Ana Bernardino & Carlos Falcão \\
Ana Cortesão Costa & Carlos Lozoya-lbáñez \\
Ana Luísa Almeida & Carlota Quintal \\
Ana Mafalda Lavrador de Jesus Carva- & Carolina Pestana \\
Iheiro & Cecília Castro \\
Ana Maria Cruz França & Celso Marialva \\
Ana Paula Vilas & Clara Vaz Marecos \\
Ana Pires Gonçalves & Cláudia Agostinho \\
Ana Povo & Cristiano Marta Figueiredo \\
Ana Raquel Fernandes & Cristina Martins Halpern \\
Ana Rita Goes & Cristina Ribeiro \\
Ana Teresa Reisinho & Cristina Valente \\
Anabela Afonso & Daniel Ribeiro Meireles \\
André Filipe Carrão & Daniel Silva Coutinho \\
Andreia Sofia Costa Teixeira & Dário Ferreira \\
António Banhudo & David Peres \\
António Jorge Cabral & Dina Gaspar \\
António Miguel Ferreira & Dina Salvador \\
António Ramalho Mostardinha & Dinarte Nuno Viveiros \\
Armandina do Carmo Antunes & Dora Margarida Leal \\
Bárbara Flor de Lima & Dulce Maria Oliveira Gomes \\
& \\
\hline &
\end{tabular}


João Gama Marques

João Melo Alves

Joaquim Varandas

Jorge Seixas

José António Ferraz Gonçalves

Judite Gonçalves

Lara Caeiro

Leandro Almeida

Leonor Moniz Pereira

Leonor Reis Boto

Luís Brandão

Luis Carreto

Luís Filipe Antunes

Luís Silva

Luís Varandas

Manuel Gonçalves-Pinho

Manuel Rosete

Manuela França

Margarida Fonseca Cardoso

Maria Francisca Moraes-Fontes

Maria Gabriela Claro Freitas Andrade

Maria Helena Almeida

Maria Isabel Santos

Maria João Polidoro

Maria João Vidigal
Maria Laureano

Mariana Barosa

Mariana Carlos Alves

Mariana Perez Duque

Mário Martins Oliveira

Marta Martins

Mauro Vinicius Dutra Girão

Natacha Lopes dos Santos

Nelea Afanas

Nuno Almeida

Olga Matos

Orlando von Doellinger

Pascoal Moleiro

Patrícia Almeida

Paula Bruno

Paula Rosa de Aldomiro

Paulo Donato

Pedro Afonso

Pedro Alberto Escada

Pedro Brandão

Pedro Câmara Pestana

Pedro Fonte

Pedro Gaspar da Costa

Pedro Silva Cunha
Peter Rice

Raquel Vieira

Ricardo A. Afonso

Ricardo Pinto Vaz

Rita Manso Araújo

Rita Vale Rodrigues

Roberto Roncon

Rui Afonso Cernadas

Sandra Nascimento

Sandra Silva Soares

Sara Sintra

Sílvia Fraga

Sofia Cerqueira

Sofia Jordão

Sofia Ribeiro

Teresa Cardoso

Tiago Azenha Rama

Tiago Dias Domingues

Tiago Torres

Tomás Silva

Tomás Teodoro

Vera Moniz-Pereira

Yuliana Olegovna Eremina

Zaida Aguiar Sá Azeredo

Revisores com uma avaliação concluída:

$\begin{array}{ll}\text { Adelaide Costa } & \text { Anabela Lopes } \\ \text { Adriana Moutinho } & \text { Anderson Garcez } \\ \text { Albino Oliveira Maia } & \text { Andrea Sofia Dias } \\ \text { Alexandra Pereira } & \text { Andreia Leite } \\ \text { Alexandra Queirós } & \text { Antónia Campos } \\ \text { Ália dos Santos } & \text { António Andrade } \\ \text { Alice Lopes } & \text { António Bugalho } \\ \text { Amílcar Oliveira } & \text { António Cardoso Fernandes } \\ \text { Ana Beatriz Nunes } & \text { António Faria Vaz } \\ \text { Ana Calistru } & \text { António MM Henriques Carneiro } \\ \text { Ana Cristina Gomes Moscoso } & \text { António Vaz-Carneiro } \\ \text { Ana Forjaz de Lacerda } & \text { Arnaldo Figueiredo } \\ \text { Ana Isabel Maia Fontes Lebre } & \text { Bárbara Antunes } \\ \text { Ana Júlia Pedro } & \text { Bárbara Marques } \\ \text { Ana Luísa Areia } & \text { Bárbara Peleteiro } \\ \text { Ana Margarida Amorim } & \text { Bernardo Marques da Silva } \\ \text { Ana Margarida Póvoa } & \text { Bruno Oliveira } \\ \text { Ana Monteiro } & \text { Bruno Rocha } \\ \text { Ana Morête } & \text { Cândida Abreu } \\ \text { Ana Nunes Barata } & \text { Carla Rodrigues } \\ \text { Ana Paula Martins } & \text { Carlos Andrade } \\ \text { Ana Paula Rocha } & \text { Carlos Silva } \\ \text { Ana R. Barbosa } & \text { Carolina Estefânia Figueira Rodrigues } \\ \text { Ana Raquel Rodrigues } & \text { Carolina Lemos } \\ \text { Ana Rita Silva } & \text { Carolina Vidal } \\ \text { Ana Sousa Menezes } & \text { Catarina Freitas } \\ \text { Ana Valério } & \text { Catarina Pereira Oliveira } \\ \text { Ana Vieira Marques } & \text { Catarina Santos de Sousa } \\ \text { Anabela Barcelos } & \text { Catarina Saraiva } \\ \text { Anabela Carocha Pinto } & \text { Célia Coelho Henriques } \\ & \end{array}$

Cidália Pina-Vaz

Cláudia Andrade

Cláudia Barroso

Claudia Costa

Cláudia Marília Rodrigues

Constantino Thedor Sakellarides

Cristiana Marinho Soares

Cristina Amaro

Cristina Furtado

Dalila Veiga

Daniel Caldeira

Daniel Macedo

Daniel Pinto

Daniela Carvalho

Daniela Carvalho

Davide Costa Carvalho

Diana Póvoas

Dinis Pestana

Diogo Teixeira

Doug McWhinnie

Duarte da Silva Soares

Duarte Miguel Rosa

Duarte Pedro de Sousa Tavares

Duarte Rego

Edgar Almeida

Eduardo Breda

Eduardo Manuel Carqueja

Eduardo Neubarth Trindade

Elicha Fernandes

Elisangela Gisele Carmo 
Elsa Landim

Elza Tomaz

Eurico Castro Alves

Eva Martins da Conceição

Fábia Cruz

Fábio Videira Santos

Fátima Cerqueira

Fátima Falcão

Fátima Serrano

Fernando Guerra

Fernando Leal da Costa

Fernando Maltez

Filipa Rocha

Filipa Sofia Silva

Filipa Teixeira

Filipe Antunes

Filipe Palavra

Filipe Peste Martinho

Filomena Azevedo

Francisco Ferreira da Silva

Frederico Soares Regateiro

Giovani Loiola Silva

Glória Cunha Velho

Gonçalo Cruz Marau

Graciete Bragança

Guilherme Gonçalves

Guilhermina Cantinho

Gustavo Cordeiro Santo

Gustavo Nobre de Jesus

Hélder Mota Filipe

Hipólito Nzwalo

Ilda Massano Cardoso

Inês Balacó

Inês Gante

Inês Palma Reis

Inês Ribeiro Vaz

Isabel Aldir

Isabel Fernandes

Isabel José de Sousa

Isabel Maria Silva Castro

Isabel Marques Brandão

Isabel Pazos

Isabel Silva

J.M. Caldas Almeida

Joana Barros Mourão

Joana Branco

Joana Branco Revés

Joana Carreteiro

Joana Esteves Martins

Joana Gomes Moscoso

Joana Ip

Joana Lopes

Joana Lopes Almeida

Joana Moreira Barros

Joana Moura Ferreira

Joana Oliveira

Joana Ramos Lopes
Joana Ribeiro dos Santos Bernardeco

Joana Rodrigues Barbosa

Joana Santos Jerónimo

Joana Verdelho Andrade

João Anselmo

João Borges Costa

João Carlos Ribeiro

João Coelho da Silva

João Filipe Raposo

João Moreira Pinto

João Nuno Ramos

João Pedro Moreira de Sousa

João Relvas

João Sérgio Neves

João Subtil

Joaquim Andrade-Gomes

Jorge Costa Santos

Jorge Moreno Governa

José Aguiar

José Alberto Pereira

José António Mariz

José Carlos Vidoedo

José Daniel Rodrigues

José Fernando Neves Santos

José Inácio Guerra Fragata

José Miguel Raimundo

José Pereira

Júlia Galhardo

Julian Perelman

Larissa Lima de Morais

Leonor de Castro Ferreira

Leonor Pinto Amaro

Lino Gonçalves

Luís Albuquerque

Luís Cabral

Luís Miguel Monteiro

Luís Pereira Amaral

Luis Roque Reis

Luís Soares Almeida

Luís Sousa

Luís Trindade

Luís Vale

Luísa Azevedo

Luiz Miguel Santiago

Madalena Sanches

Mafalda Felgueiras

Mafalda Seabra

Manuel Gonçalves-Pereira

Manuel João Brito

Manuel Luís Vila Capelas

Manuel Mendes Silva

Manuel P. Morgado

Manuel Rodrigues Pereira

Manuela Carvalheiro

Margarida Abrantes

Margarida Figueiredo Dias

Margarida Maria Marques
Margarida Pimenta Valério

Margarida Pocinho

Margarida Silvestre

Maria Arminda Guilherme

Maria Clara Pedro Bicho

Maria do Carmo Vale

Maria Fernanda Diamantino

Maria Isabel Correia Dias

Maria João Rocha Brito

Maria João Simões

Maria José Santos

Maria Raquel Carvalho

Maria Salomé Carvalho

Mariana Alves

Mariana Amaral

Mariana Freitas Miranda

Mariana Poppe

Marina Pinheiro

Mário Fontes e Sousa

Mário Marques Vieira

Marta Alves

Marta Carvalho

Marta Ferreira

Marta Zegre Amorim

Mesquita Bastos

Miguel Bajouco

Miguel Gouveia

Mourão Carvalho

Natalia Marto

Nelson Fernando Gomes Oliveira

Nídia Zozimo

Noémia Afonso

Nuno Alegrete

Nuno Figueiredo

Nuno Pereira

Nuno Rocha

Pablo De Castro Santos

Patrícia Afonso Mendes

Patrícia Alves

Patrícia José

Paulo Camargos

Paulo Nascimento Simoes

Paulo Reis-Pina

Paulo Santos

Paulo Varela

Pedro Andrade

Pedro Coelho

Pedro Filipe Sousa

Pedro Gardete

Pedro Magalhães Oliveira

Pedro Melvill de Araújo

Pedro Mesquita Oliveira

Pedro Morgado

Pedro Mota Veiga

Pedro Narra Figueiredo

Quirina Santos-Costa

Raquel Duarte 
Raquel Faria

Raquel Fernandes Castro

Regina Ferreira Alves

Ricardo Dias Coelho

Rita Araújo

Rita Gomes

Rita Marques

Rodrigo Santos Vicente

Rosete Rosete Nogueira

Rui Alves

Rui António da Cruz de Vasconcellos

Guimarães

Rui Barranha

Rui Barreto
Rui Bello

Rui Miguel Martins

Rui Morais

Rui Rolo

S.M. Yasir Arafat

Sara Donato

Silvia Álvares

Sílvio Bollini

Susana Archer Carvalho

Susana Gomes

Tabita Magalhães Maia

Telma Barbosa

Teresa Goldschmidt

Teresa M Salgado
Teresa Seara Sevivas

Tiago Bilhim

Tiago da Silva Santos

Tiago Filipe Ferreira

Tiago Fonseca

Tiago M Alfaro

Vasco Gil Calado

Vasco Ricoca Peixoto

Vera Afreixo

Vera Trocado

Virgínia Monteiro

Zélia Gomes 